\title{
Microglial activation, but not tau pathology, is independently associated with amyloid positivity and memory impairment
}

\author{
James Zou ${ }^{\mathrm{a}, *}$, Sha Tao ${ }^{\mathrm{b}}$, Aubrey Johnson ${ }^{\mathrm{a}}$, Zeljko Tomljanovic ${ }^{\mathrm{a}}$, Krista Polly ${ }^{\mathrm{a}}$, \\ Julia Klein ${ }^{a}$, Qolamreza R. Razlighi ${ }^{a}$, Adam M. Brickman ${ }^{a}$, Seonjoo Lee ${ }^{b}$, \\ Yaakov Stern ${ }^{\text {a }}$, William Charles Kreisl ${ }^{\text {a }}$

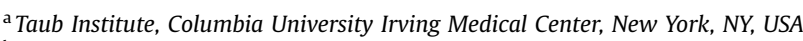 \\ ${ }^{\mathrm{b}}$ Mailman School of Public Health, Columbia University Irving Medical Center, New York, NY, USA
}

\section{A R T I C L E I N F O}

\section{Article history:}

Received 9 May 2019

Received in revised form 22 August 2019

Accepted 25 September 2019

Available online 29 September 2019

\section{Keywords:}

Alzheimer's disease

Amyloid

Tau

Microglia

Neuroinflammation

\begin{abstract}
A B S T R A C T
We sought to determine if upstream amyloid accumulation and downstream cognitive impairment have independent relationships with microglial activation and tau pathology. Fifty-eight older adults were stratified by amyloid and cognitive status based on ${ }^{18} \mathrm{~F}$-florbetaben PET, history, and neuropsychological testing. Of these, 57 had ${ }^{11} \mathrm{C}$-PBR28 PET to measure microglial activation and 43 had ${ }^{18} \mathrm{~F}$-MK-6240 PET to measure tau pathology. Amyloid and cognitive status were associated with increased overall binding for both ${ }^{11} \mathrm{C}$-PBR28 and ${ }^{18} \mathrm{~F}-\mathrm{MK}-6240$ ( $p$ 's $<0.01$ ). While there was no interaction between amyloid and cognitive status in their association with ${ }^{11} \mathrm{C}$-PBR28 binding $(p=0.6722)$, there was an interaction in their association with ${ }^{18} \mathrm{~F}-\mathrm{MK}-6240$ binding $(p=0.0115)$. Binding of both radioligands was greater in amyloid-positive controls than in amyloid-negative controls; however, this difference was seen in neocortical regions for ${ }^{11} \mathrm{C}$-PBR28 and only in medial temporal cortex for ${ }^{18} \mathrm{~F}-\mathrm{MK}-6240$. We conclude that, in the absence of cognitive symptoms, amyloid deposition has a greater association with microglial activation than with tau pathology.
\end{abstract}

(c) 2019 Elsevier Inc. All rights reserved.

\section{Introduction}

The emergence of $\beta$-amyloid pathophysiology-defined by reduction of $A \beta_{42}$ in CSF or by cortical retention of amyloid radioligands on PET-is the earliest detectable in vivo change in Alzheimer's disease (AD) pathogenesis (Jack et al., 2013). However, imaging studies have identified both cognitive controls with high amyloid signal and patients with Alzheimer's-like impairment with no apparent amyloid binding (Landau et al., 2016; Petersen et al., 2013; Pike et al., 2011), demonstrating that amyloidosis is neither necessary nor sufficient for clinical expression of an Alzheimer's phenotype. Therefore, other pathogenic factors appear to be required for cognitive decline, in both $\mathrm{AD}$ and in disorders that mimic AD.

Neuroinflammation is related to cognitive decline in AD. Binding of PET radioligands to the $18 \mathrm{kDa}$ translocator protein (TSPO) - a marker of microglial activation-is increased in AD and

\footnotetext{
* Corresponding author at: Taub Institute, Columbia University Irving Medical Center, 622 W. 168th Street, PH Building 19th Floor, New York, NY 10032, USA. Tel.: 408-768-0258; fax: 212-305-2526.

E-mail address: jsz2108@cumc.columbia.edu (J. Zou).
}

correlates with disease severity (Edison et al., 2008; Kreisl et al., 2013b, 2016). However, neuroinflammation may be a nonspecific response to neurodegeneration, as TSPO binding is increased in several non-Alzheimer neurodegenerative disorders, including frontotemporal dementia, Parkinson's disease, and amyotrophic lateral sclerosis (Cagnin et al., 2004; Corcia et al., 2012; Gerhard et al., 2006), and in vitro studies demonstrate microglial localization to axonal debris (Tanaka et al., 2009). On the other hand, recent genome-wide association studies identified mutations in microglia-expressed genes (e.g., TREM2 and CD33) that confer increased risk of AD (Colonna and Wang, 2016). Moreover, results from tissue culture studies showed that physiological concentrations of $A \beta_{42}$ stimulate microglia to release neurotoxic cytokines (Maezawa et al., 2011; Marlatt et al., 2014), and inflammatory markers such as YKL-40 are elevated in CSF in the preclinical and prodromal stages of AD (Janelidze et al., 2018). These latter results suggest that microglial activation appears early in AD pathogenesis, perhaps in response to early amyloid deposition, and may contribute to clinical progression.

PET imaging studies show that neurofibrillary aggregation of tau is more frequent in amyloid-positive than amyloid-negative controls (Jack et al., 2018; Schultz et al., 2018), and tau pathology is 
generally detected after substantial amyloid plaque deposition (Jack et al., 2013). To date, only 2 PET studies have measured these 3 biomarkers in the same subjects. One study found correlations between TSPO and tau binding in both mild cognitive impairment and AD patients (Dani et al., 2018); however, correlations were also found in amyloid-negative subjects and the measured amount of TSPO binding was similar among patients and controls. The other study found no correlation between TSPO and tau binding (Parbo et al., 2018). This latter study may have lacked sensitivity though, because of the low specific-to-nonspecific binding of the TSPO radioligand used, ${ }^{11} \mathrm{C}-(R)-\mathrm{PK} 11195$.

We sought to determine how microglial activation and tau pathology relate to the upstream amyloidosis and downstream cognitive decline seen in AD. To achieve this goal, we stratified subjects by amyloid and cognitive status to test if amyloid-positivity and cognitive impairment are each independently associated with the amount of microglial activation and tau pathology measured with PET. We also sought to infer a temporal order of these Alzheimer's-associated markers from the cross-sectional imaging data. Microglial activation was measured using the TSPO radioligand ${ }^{11} \mathrm{C}$ PBR28 (Briard et al., 2008; Lyoo et al., 2015). Tau pathology was measured with ${ }^{18} \mathrm{~F}-\mathrm{MK}-6240$, a recently developed radioligand that binds to paired helical filament tangles (Betthauser et al., 2019; Hostetler et al., 2016; Lohith et al., 2019; Pascoal et al., 2018).

\section{Materials and methods}

\subsection{Subject selection}

Seventy-three adults aged 50 years and older were recruited from the Columbia University Irving Medical Center (CUIMC) Aging and Dementia clinic, self-referrals, the Columbia Alzheimer's Disease Research Center, and other research cohorts at CUIMC. All subjects underwent routine history, physical and neurological examination; routine laboratory studies; and a T1 MPRAGE (160 slice $1 \mathrm{~mm}$ resolution, $256 \times 200$ voxel count) on a 3T Phillips Achieva MRI machine in the CUIMC Hatch MRI center.

These screening procedures were performed to rule out significant medical, neurological, or psychiatric illness; presence of cortical infarcts on imaging; and use of any immunosuppressive medication. All subjects (or their representative) provided informed consent according to the Declaration of Helsinki and this study was approved by the CUIMC Institutional Review Board.

\subsection{Cognitive testing}

Subjects who passed screening underwent neuropsychological testing, which included the Clinical Dementia Rating scale (Hughes et al., 1982), Mini-Mental State Examination (MMSE) (Folstein et al., 1983), Selective Reminding Test Delayed Recall (Ruff et al., 1989), Trail Making Test Parts A and B, and Category and Phonemic Fluency. All cognitive test scores were transformed into z-scores using age-, sex-, and education-adjusted normative data. To be considered cognitively impaired, subjects had to have a primary memory complaint and meet clinical criteria for either amnestic mild cognitive impairment (MCI) (Albert et al., 2011) or AD (McKhann et al., 2011). None of the impaired patients met clinical criteria for any specific disorder other than AD (e.g., dementia with Lewy bodies, vascular dementia, Parkinson's disease dementia, corticobasal degeneration, progressive supranuclear palsy, or frontotemporal dementia). Control subjects were free of any cognitive complaint, and history and neuropsychological test scores were used to confirm absence of clinically meaningful cognitive impairment.

\subsection{TSPO genotyping}

TSPO binding affinity was determined at screening. Genomic DNA from each subject was used to genotype the rs6971 polymorphism using a TaqMan assay (Owen et al., 2012). Subjects homozygous for the low affinity allele were excluded from any further testing detailed in the following, and from the final analysis $(n=8)$.

\subsection{PET imaging}

All PET imaging was performed in the CUIMC Kreitchman PET Center on the same Siemens Biograph mCT with low-dose CT scan used for attenuation correction. PET scans were performed on separate days. Amyloid status was determined with ${ }^{18} \mathrm{~F}$-florbetaben (FBB) PET. FBB images were acquired from 50-70 minutes after injection (injected activity $=300 \mathrm{MBq}$ ) in $4 \times 5$ minutes frames. ${ }^{11} \mathrm{C}$ PBR28 PET images were acquired 60-90 minutes after injection (injected activity $=599 \pm 140 \mathrm{MBq}$ ) in $6 \times 5$ minutes frames. ${ }^{18} \mathrm{~F}$ MK-6240 images were acquired 80-100 minutes after injection (injected activity $=172 \pm 10 \mathrm{MBq}$ ) in $4 \times 5$ minutes frames. All ${ }^{11} \mathrm{C}$ PBR28 scans were performed within 12 months of MRI and FBB scan. Mean interval between ${ }^{11}$ C-PBR28 and ${ }^{18} \mathrm{~F}-\mathrm{MK}-6240$ scans was $6 \pm 4$ months for patients and $11 \pm 7$ months for controls. Subjects who had greater than 12 months delay between ${ }^{11} \mathrm{C}$-PBR28 and ${ }^{18} \mathrm{~F}-\mathrm{MK}-6240$ scans had neuropsychological testing and brain MRI repeated.

\subsubsection{PET image processing}

The FBB, ${ }^{11} \mathrm{C}-\mathrm{PBR} 28$, and ${ }^{18} \mathrm{~F}-\mathrm{MK}-6240$ PET images were processed using the same pipeline. Reconstructed PET images were first realigned and corrected for subject motion using SPM12 (Wellcome Centre for Human Neuroimaging.). Images were then preprocessed with PMOD 3.8 (PMOD Technologies) using the PNEURO tool. T1-weighted MRI scans were segmented and normalized to standard space and the Hammers-N30R83-1 MM atlas was used to define regions of interest (ROIs). The ROI volumes were then reverse-warped to the subject's native space. ROIs were inspected and manually corrected as necessary. PET images were coregistered to the native space MRI. For ${ }^{11} \mathrm{C}$-PBR2 8 and ${ }^{18} \mathrm{~F}$ MK-6240, the coregistered PET images were then corrected for partial volume effects with the region-based voxelwise method (Thomas et al., 2011). PET images from each acquisition (50-70 minutes for FBB, 60-90 minutes for ${ }^{11} \mathrm{C}-\mathrm{PBR} 28$, 80-100 minutes for ${ }^{18} \mathrm{~F}-\mathrm{MK}-6240$ ) were then averaged, and the native space ROIs were applied. Concentration of radioactivity $(\mathrm{kBq} / \mathrm{cc})$ was then extracted from each ROI.

Volume-weighted gray matter ROIs were created from the Hammers atlas ROIs, consisting of prefrontal cortex (middle frontal gyrus, inferior frontal gyrus, superior frontal gyrus, straight gyrus, anterior orbital gyrus, medial orbital gyrus, lateral orbital gyrus, posterior orbital gyrus); middle and inferior temporal gyri (medial part of anterior temporal lobe, lateral parts of anterior temporal lobe and middle and inferior temporal gyri); superior temporal gyrus (anterior part of superior temporal gyrus, posterior part of superior temporal gyrus); medial temporal cortex (amygdala, parahippocampal gyrus, and entorhinal cortex); posterior cingulate cortex; superior parietal lobule; inferior parietal lobule; striatum (caudate nucleus and putamen); and cerebellum. For FBB and ${ }^{11} \mathrm{C}$ PBR28, the concentration of radioactivity of each ROI was then divided by that of the cerebellum to create standardized uptake value ratios (SUVRs), a method that has been validated for both tracers in prior studies (Bullich et al., 2017; Lyoo et al., 2015). Because uptake of ${ }^{18} \mathrm{~F}-\mathrm{MK}-6240$ is sometimes seen in the anterior lobe of the cerebellum (Betthauser et al., 2019), likely due to spillover from ventral temporal cortex and occipital cortex or off- 
target binding in tentorium cerebelli, we used only inferior cerebellar gray matter as reference region for this radioligand. For ${ }^{11} \mathrm{C}$ PBR28 and ${ }^{18} \mathrm{~F}-\mathrm{MK}-6240$, SUVRs were calculated for both partial volume-corrected and uncorrected images.

\subsubsection{Amyloid status determination}

Reconstructed FBB PET images were averaged to create a single image for each subject and a visual read by an experienced neurologist (WCK) blinded to the subject's diagnosis was used to determine the presence or absence of fibrillar amyloid plaque (Bullich et al., 2017). ROI data from the 50-70 minutes FBB images were used to calculate a composite SUVR (weighted average of SUVRs from prefrontal cortex, middle, and inferior temporal gyri, superior temporal gyrus, medial temporal cortex, posterior cingulate cortex, superior parietal lobule, and inferior parietal lobule) using cerebellar gray matter as reference region.

\subsubsection{T1 MRI analysis}

Volumes for target ROIs (prefrontal cortex, middle, and inferior temporal gyrus, superior temporal gyrus, medial temporal cortex, posterior cingulate cortex, superior parietal lobule, inferior parietal lobule, striatum, and cerebellum) were derived using PMOD as detailed in 2.4.1. To determine if amyloid-negative patients had an Alzheimer's-like pattern of neurodegeneration, ROIs for the right and left hippocampi for all subjects were manually drawn by investigators blind to the diagnosis. The volume of each ROI was corrected for differences in brain size by dividing by the total intracranial volume.

\subsection{Statistical analysis}

Statistical analyses were carried out using SAS version 9.4 and $R$ version 3.5.1. Subjects were classified based on their amyloid status and clinical profile (as described in 2.2 and 2.4.2) into one of 4 groups: (1) amyloid-positive patients, (2) amyloid-positive controls, (3) amyloid-negative patients, and (4) amyloid-negative controls. Mean and standard deviation was derived for key descriptive characteristics for each subgroup, and an analysis of variance was performed to assess between group differences in these descriptive statistics. To validate the visual read of amyloid status as described in 2.4.2, the composite FBB SUVR was compared between amyloid-positive and amyloid-negative subjects.
To determine if amyloid and cognitive status are related to microglial activation and pathological tau burden, we performed separate multivariate analyses of variance (MANOVA) for ${ }^{11} \mathrm{C}-\mathrm{PBR} 28$ and ${ }^{18} \mathrm{~F}-\mathrm{MK}-6240$ binding, using the 8 PMOD-derived target ROIs: prefrontal cortex; middle and inferior temporal gyri; superior temporal gyrus; medial temporal cortex; posterior cingulate; superior parietal lobule; inferior parietal lobule; and striatum. Amyloid and cognitive status were the independent variables. Age and TSPO genotype (for ${ }^{11} \mathrm{C}$-PBR28 binding) were included in the model as covariates. We also tested for interactions between amyloid positivity and cognitive impairment. For all MANOVA analyses, the residuals were examined to check normality and sphericity.

To more closely determine if amyloid and cognitive status are related to microglial activation and tau burden on a regional level, we ran a univariate analysis of variance for each of the 8 target ROIs for both ${ }^{11} \mathrm{C}$-PBR2 8 and ${ }^{18} \mathrm{~F}-\mathrm{MK}-6240$ binding. Amyloid and cognitive status were the independent variables. Age and TSPO genotype (for ${ }^{11} \mathrm{C}$-PBR28 binding) were included as covariates. We determined effect size using partial Eta-square and tested for interactions between amyloid and cognitive status. For this primary analysis, we applied Bonferroni correction to compensate for the 16 multiple comparisons ( 2 independent variables $\times 8$ regions).

In a secondary analysis, to determine when and where ${ }^{11} \mathrm{C}$ PBR28 and ${ }^{18} \mathrm{~F}-\mathrm{MK}-6240$ binding first increase, we stratified subjects along the $\mathrm{AD}$ clinical spectrum from normal aging to $\mathrm{AD}$ dementia (i.e., amyloid-negative controls, amyloid-positive controls, amyloid-positive patients with mild cognitive impairment, and amyloid-positive patients with dementia), and then performed a four-way ANOVA to test for between-group differences within each of the 8 aforementioned ROIs. Partial correlations, adjusting for age and TSPO genotype (for ${ }^{11} \mathrm{C}$-PBR28), tested the relationships of MMSE score with ${ }^{11} \mathrm{C}$-PBR28 and ${ }^{18} \mathrm{~F}-\mathrm{MK}-6240$ binding in these ROIs as well. Partial correlations were also performed to test the relationship between ${ }^{11} \mathrm{C}$-PBR2 8 and ${ }^{18} \mathrm{~F}-\mathrm{MK}$ 6240 binding in each region.

\section{Results}

\subsection{Characteristics of included participants}

Fifty-seven subjects completed screening procedures, MRI, and the ${ }^{11}$ C-PBR28 scan: 23 amyloid-positive patients, 7 amyloid-

Table 1

Descriptive data for subject participants based on amyloid and cognitive status ${ }^{\mathrm{a}}$

\begin{tabular}{|c|c|c|c|c|c|c|c|}
\hline Variable & $\begin{array}{l}\mathrm{A} \beta(+) \text { patients } \\
(\mathrm{n}=23)\end{array}$ & $\begin{array}{l}\mathrm{A} \beta(+) \text { controls } \\
(\mathrm{n}=7)\end{array}$ & $\begin{array}{l}\mathrm{A} \beta(-) \text { patients } \\
(\mathrm{n}=10)\end{array}$ & $\begin{array}{l}\mathrm{A} \beta(-) \text { controls } \\
(\mathrm{n}=17)\end{array}$ & $\begin{array}{l}\text { F Statistics of } \\
\text { 4-group ANOVA }\end{array}$ & $\begin{array}{l}p \text {-value of } F \\
\text { statistics }\end{array}$ & $\begin{array}{l}\text { Partial } \\
\text { eta-square }\end{array}$ \\
\hline Age $(y)^{b, c, g}$ & $65.8 \pm 8.4$ & $73.1 \pm 2.7$ & $75.8 \pm 9.8$ & $67.6 \pm 3.8$ & 5.87 & 0.0015 & 0.2459 \\
\hline Male/Female & $20 / 3$ & $4 / 3$ & $7 / 4$ & $5 / 12$ & $\mathrm{~N} / \mathrm{A}$ & $\mathrm{N} / \mathrm{A}$ & $\mathrm{N} / \mathrm{A}$ \\
\hline Education (y) & $16.3 \pm 2.7$ & $14.9 \pm 2.5$ & $16.5 \pm 3.5$ & $15.6 \pm 2.7$ & 0.66 & 0.5804 & 0.0354 \\
\hline MMSE score ${ }^{\mathrm{b}, c, \mathrm{~d}}$ & $23.0 \pm 4.7$ & $28.9 \pm 2.0$ & $26.9 \pm 2.8$ & $29.4 \pm 0.8$ & 14.19 & $<0.0001$ & 0.4407 \\
\hline SRT-DR (z-score) $)^{\mathrm{b}, \mathrm{c}, \mathrm{d}, \mathrm{e}, \mathrm{g}}$ & $-3.32 \pm 0.45$ & $0.23 \pm 1.19$ & $-2.53 \pm 0.63$ & $0.72 \pm 1.02$ & 100.95 & $<0.0001$ & 0.8511 \\
\hline TSPO genotype (HAB/MAB) & $13 / 10$ & $3 / 4$ & $6 / 5$ & $12 / 5$ & $\mathrm{~N} / \mathrm{A}$ & N/A & $\mathrm{N} / \mathrm{A}$ \\
\hline FBB Composite SUVR ${ }^{\mathrm{b}, c, \mathrm{~d}, \mathrm{e}, \mathrm{f}}$ & $1.65 \pm 0.18$ & $1.49 \pm 0.14$ & $1.17 \pm 0.14$ & $1.12 \pm 0.06$ & 54.04 & $<0.0001$ & 0.7501 \\
\hline \% Hippocampal Volume ${ }^{\mathrm{b}, \mathrm{d}, \mathrm{g}}$ & $0.86 \pm 0.15$ & $1.00 \pm 0.16$ & $0.85 \pm 0.19$ & $1.04 \pm 0.14$ & 5.69 & 0.0018 & 0.2402 \\
\hline \% Inferior Parietal Lobule ${ }^{b, c, d}$ & $5.19 \pm 0.41$ & $5.66 \pm 0.34$ & $5.73 \pm 0.24$ & $5.61 \pm 0.40$ & 7.46 & 0.0003 & 0.2929 \\
\hline Global WMH score $\left(\mathrm{mm}^{3}\right)^{g}$ & $2.33 \pm 2.03$ & $4.85 \pm 5.75$ & $4.58 \pm 4.76$ & $1.78 \pm 2.80$ & 2.38 & 0.0795 & 0.1189 \\
\hline
\end{tabular}

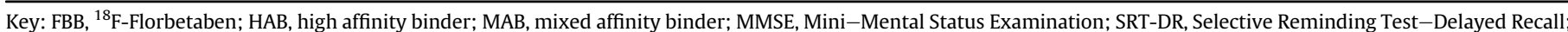
SUVR, standardized uptake value ratio; TSPO, $18 \mathrm{kDa}$ translocator protein; \% volume, \% total intracranial volume; WMH, white matter hyperintensity.

${ }^{a}$ Fifteen participants did not undergo ${ }^{18} \mathrm{~F}-\mathrm{MK}-6240$ PET and one participant did not undergo ${ }^{11} \mathrm{C}$-PBR28 PET.

b Significant difference $(p<0.05)$ between $A \beta(+)$ patients and $A \beta(+)$ controls.

c Significant difference $(p<0.05)$ between $A \beta(+)$ patients and $A \beta(-)$ patients.

d Significant difference $(p<0.05)$ between $A \beta(+)$ patients and $A \beta(-)$ controls.

e Significant difference $(p<0.05)$ between $A \beta(+)$ controls and $A \beta(-)$ patients.

f Significant difference $(p<0.05)$ between $A \beta(+)$ controls and $A \beta(-)$ controls.

g Significant difference $(p<0.05)$ between $A \beta(-)$ patients and $A \beta(-)$ controls. 
Table 2

Partial volume-corrected ${ }^{11}$ C-PBR28 SUVR values for each subject group, stratified by amyloid and cognitive status

\begin{tabular}{|c|c|c|c|c|c|c|c|c|c|c|}
\hline \multirow[t]{2}{*}{ Region } & \multirow{2}{*}{$\begin{array}{l}\mathrm{A} \beta(+) \text { patients } \\
(\mathrm{n}=23)\end{array}$} & \multirow{2}{*}{$\begin{array}{l}\mathrm{A} \beta(+) \text { controls } \\
(\mathrm{n}=7)\end{array}$} & \multirow{2}{*}{$\begin{array}{l}A \beta(-) \text { patients } \\
(n=10)\end{array}$} & \multirow{2}{*}{$\begin{array}{l}A \beta(-) \text { controls } \\
(n=17)\end{array}$} & \multicolumn{3}{|l|}{$\mathrm{A} \beta$ status } & \multicolumn{3}{|l|}{ Cognitive status } \\
\hline & & & & & F Statistic $_{(1,52)}$ & $p$-value & $\begin{array}{l}\text { Partial } \\
\text { eta squared }\end{array}$ & F Statistic $_{(1,52)}$ & $p$-value & $\begin{array}{l}\text { Partial } \\
\text { eta squared }\end{array}$ \\
\hline Prefrontal & $1.27 \pm 0.08$ & $1.21 \pm 0.07$ & $1.22 \pm 0.15$ & $1.10 \pm 0.17$ & 6.32 & 0.0151 & 0.1083 & 7.80 & 0.0073 & 0.1305 \\
\hline $\begin{array}{l}\text { Mid/Inf } \\
\text { temporal }\end{array}$ & $1.24 \pm 0.13$ & $1.13 \pm 0.06$ & $1.17 \pm 0.13$ & $1.04 \pm 0.10$ & 7.51 & 0.0084 & 0.1262 & 14.86 & $0.0003^{\mathrm{a}}$ & 0.2223 \\
\hline $\begin{array}{l}\text { Superior } \\
\text { temporal }\end{array}$ & $1.23 \pm 0.08$ & $1.19 \pm 0.07$ & $1.18 \pm 0.15$ & $1.09 \pm 0.13$ & 7.09 & 0.0103 & 0.1200 & 3.93 & 0.0528 & 0.0702 \\
\hline $\begin{array}{l}\text { Medial } \\
\text { temporal }\end{array}$ & $1.25 \pm 0.18$ & $1.15 \pm 0.16$ & $1.16 \pm 0.16$ & $1.02 \pm 0.13$ & 7.13 & 0.0101 & 0.1206 & 9.24 & 0.0037 & 0.1508 \\
\hline $\begin{array}{l}\text { Posterior } \\
\text { cingulate }\end{array}$ & $1.27 \pm 0.11$ & $1.13 \pm 0.08$ & $1.16 \pm 0.17$ & $1.09 \pm 0.12$ & 7.08 & 0.0103 & 0.1199 & 10.24 & $0.0023^{\mathrm{a}}$ & 0.1645 \\
\hline $\begin{array}{l}\text { Superior } \\
\text { parietal }\end{array}$ & $1.29 \pm 0.12$ & $1.16 \pm 0.11$ & $1.14 \pm 0.14$ & $1.04 \pm 0.16$ & 14.52 & $0.0004^{a}$ & 0.2183 & 9.78 & $0.0029^{\mathrm{a}}$ & 0.1582 \\
\hline $\begin{array}{l}\text { Inferior } \\
\text { parietal }\end{array}$ & $1.25 \pm 0.11$ & $1.11 \pm 0.08$ & $1.11 \pm 0.13$ & $1.00 \pm 0.15$ & 12.50 & $0.0009^{\mathrm{a}}$ & 0.1938 & 11.49 & $0.0013^{\mathrm{a}}$ & 0.1809 \\
\hline Striatum & $0.85 \pm 0.08$ & $0.81 \pm 0.07$ & $0.86 \pm 0.06$ & $0.82 \pm 0.13$ & 0.00 & 0.9621 & 0.0000 & 2.72 & 0.1052 & 0.0497 \\
\hline
\end{tabular}

Key: SUVR, standardized uptake value ratio.

a Survives Bonferroni correction of 16 tests (corrected $p$-value $<0.05$ ).

positive controls, 10 amyloid-negative patients, and 17 amyloidnegative controls. Forty-two of these (14 amyloid-positive patients, 7 amyloid-positive controls, 9 amyloid-negative patients, and 12 amyloid-negative controls), plus one amyloid-negative patient who did not undergo ${ }^{11} \mathrm{C}$-PBR28 imaging, had ${ }^{18} \mathrm{~F}-\mathrm{MK} 6240$ PET. One amyloid-positive patient was unable to complete the SRT because of poor memory performance. Impaired status for this participant was assigned based on memory complaint corroborated by caregiver, $0 / 3$ delayed recall on MMSE testing, and performance on nonmemory tests. Both patient groups had lower Selective Reminding Test Delayed Recall z-scores than the control groups ( $p$ 's $<0.01$ ); however, amyloid-positive patients had lower MMSE score than the other 3 groups ( $p$ 's $<0.01$ ). Both patient groups had smaller hippocampal volume (corrected for total intracranial volume) than the amyloid-negative controls ( $p$ 's $<0.01$ ). Amyloidpositive patients additionally had smaller corrected volume for inferior parietal lobule than the other 3 groups ( $p$ 's $<0.01$ ), whereas amyloid-negative patients had smaller corrected volume for
A Middle and inferior temporal gyri

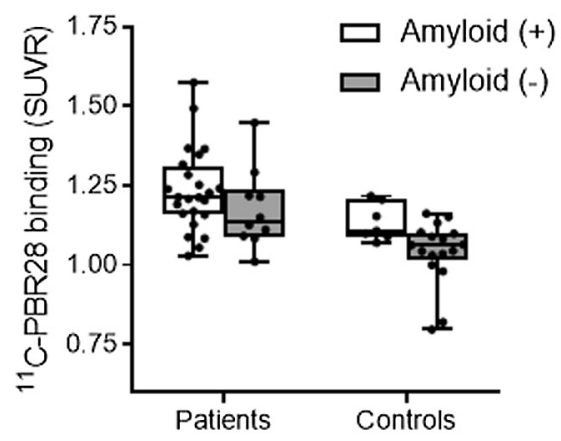

B

Middle and inferior temporal gyri

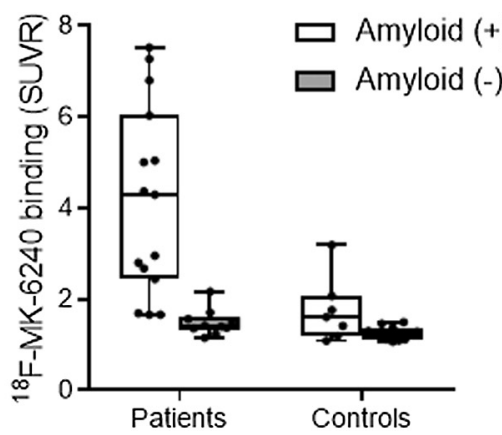

Medial temporal cortex

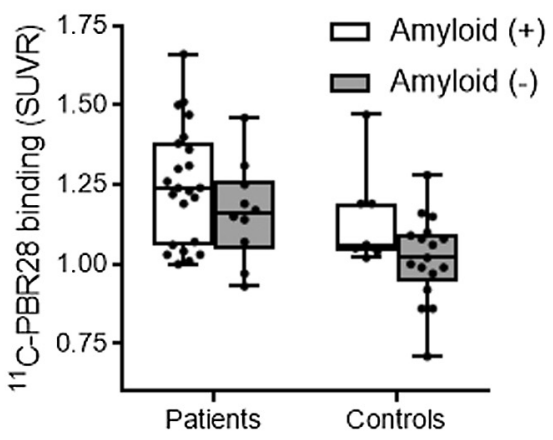

Medial temporal cortex

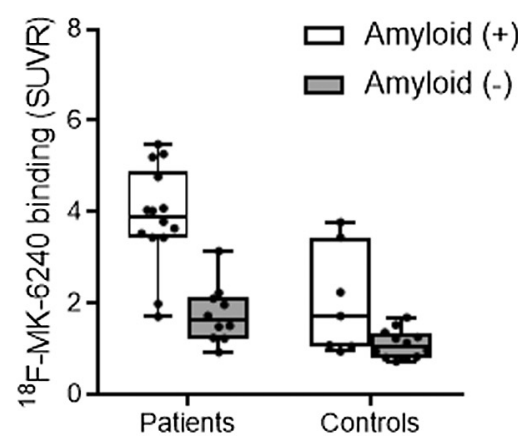

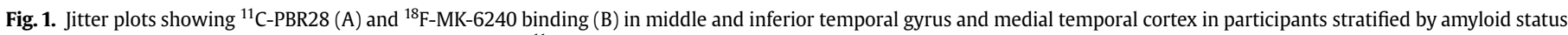

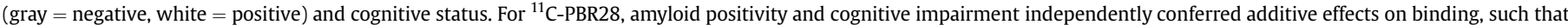

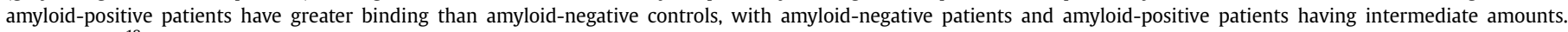
However, for ${ }^{18} \mathrm{~F}-\mathrm{MK}-6240$, greater binding is only seen in amyloid-positive patients. Abbreviations: SUVR, standardized uptake value ratio. 


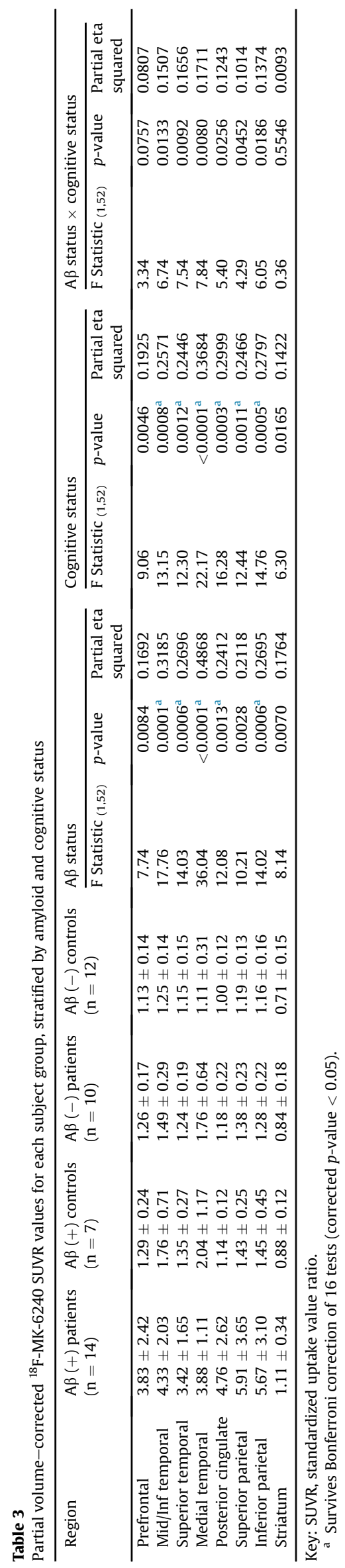

striatum than the other 3 groups ( $p$ 's $<0.01$ ). No group differences in corrected volume were seen for any other ROI. In agreement with our visual read of amyloid status, both amyloid-positive groups had greater FBB SUVRs in the composite ROI than the amyloid-negative groups ( $p$ 's $<0.01$ ). Table 1 shows demographic and clinical information for the 58 participants included in the analysis. The number of high affinity ("HAB") and mixed affinity ("MAB") binders in each group are also reported.

\subsection{Effect of amyloid status and cognitive impairment on ${ }^{11} \mathrm{C}-\mathrm{PBR} 28$ binding}

We first tested how microglial activation relates to amyloid and cognitive status. We found that amyloid positivity, cognitive impairment, greater age, and high affinity TSPO genotype all had positive associations with overall ${ }^{11} \mathrm{C}$-PBR28 binding ( $p$ 's $<0.01$ ). However, we found no interaction between amyloid status and cognitive status $(p=0.6722)$.

We then looked at regional effects of amyloid and cognitive status on microglial activation. After correcting for age and TSPO genotype, we found a positive association of both amyloid positivity and cognitive impairment on ${ }^{11} \mathrm{C}$-PBR28 binding in most target ROIs (Table 2). Amyloid positivity was associated with greater ${ }^{11} \mathrm{C}$-PBR28 binding in all regions except for the striatum. Cognitive impairment was associated with greater ${ }^{11} \mathrm{C}$-PBR28 binding in all regions except superior temporal gyrus and striatum. There were no interactions between amyloid status and cognitive status observed in any region. The independence of amyloid-positivity and cognitive impairment resulted in additive effects on ${ }^{11} \mathrm{C}$-PBR28 binding, such that amyloid-positive patients had greater binding than amyloidnegative controls, with amyloid-positive controls and amyloidnegative patients having intermediate binding (Fig 1A).

Increased age was associated with greater ${ }^{11} \mathrm{C}$-PBR28 binding in middle and inferior temporal gyri, superior temporal gyrus, and medial temporal cortex ( $p$ 's $<0.05$ ). TSPO genotype had an effect on ${ }^{11} \mathrm{C}$-PBR28 binding in middle and inferior temporal gyri, medial temporal cortex, posterior cingulate cortex, superior parietal lobule, and striatum ( $p$ 's $<0.05$ ).

The results of the analyses using ${ }^{11} \mathrm{C}$-PBR28 data uncorrected for partial volume effects generally agreed with those using partial volume-corrected data. Uncorrected SUVRs for each group are reported in Supplementary Table 1.

\subsection{Effect of amyloid status and cognitive impairment on ${ }^{18} \mathrm{~F}-\mathrm{MK}$ - 6240 binding}

When we tested how tau pathology relates to amyloid and cognitive status, we found that amyloid positivity $(p=0.0007)$ and cognitive impairment ( $p=0.0025$ ) were associated with greater ${ }^{18}$ F-MK-6240 binding. However, unlike with ${ }^{11} \mathrm{C}$-PBR28 binding, we found an overall interaction between amyloid positivity and cognitive impairment ( $p=0.0115)$. The interaction between amyloid positivity and cognitive impairment resulted in a multiplicative effect on ${ }^{18} \mathrm{~F}$-MK6240 binding, such that binding was increased in amyloid-positive patients and low in the other 3 groups (Fig 1B). There was no effect of age on ${ }^{18} \mathrm{~F}-\mathrm{MK}-6240$ binding $(p=0.2987)$.

When we looked at regional effects of amyloid and cognitive status on tau pathology, we found that both amyloid positivity and cognitive impairment were associated with greater ${ }^{18} \mathrm{~F}-\mathrm{MK}-6240$ binding in all measured ROIs ( $p$ 's $<0.05$, Table 3 ). Positive interactions between amyloid positivity and cognitive impairment were observed in all ROIs ( $p$ 's $<0.05$ ) except prefrontal cortex (although an interaction was seen here at trend level with $p=0.0757)$ and striatum $(p=0.5546)$. We found no effect of age in any ROI. 

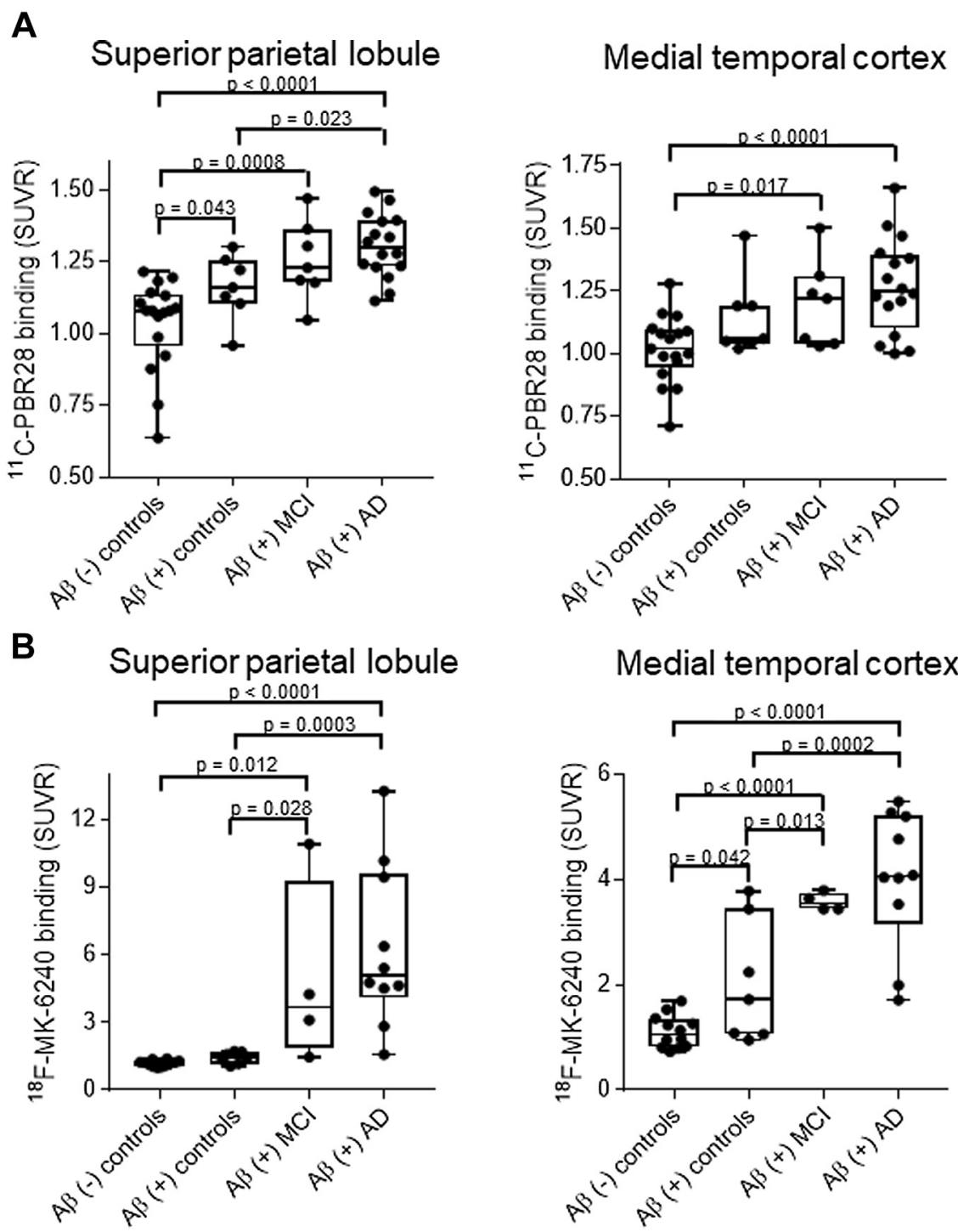

\section{Medial temporal cortex}

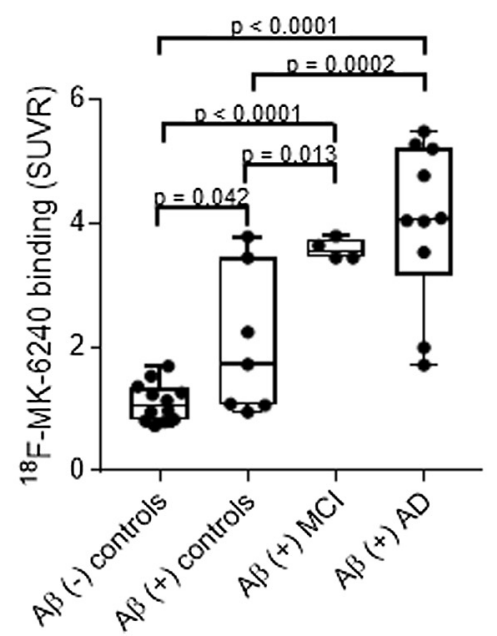

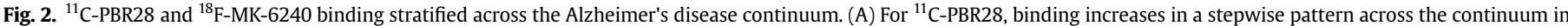

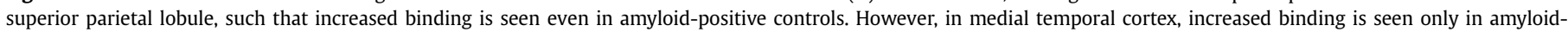

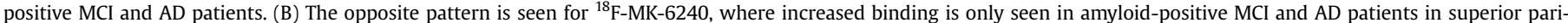

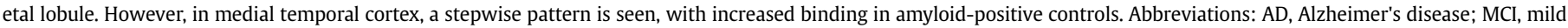
cognitive impairment; SUVR, standardized uptake value ratio.

As with ${ }^{11} \mathrm{C}$-PBR28, the results of the analyses using data uncorrected for partial volume effects generally agreed with those using partial volume-corrected data. Uncorrected SUVRs for each group are reported in Supplementary Table 2.

We corrected for MANOVAs performed (testing microglial activation and tau pathology). After multiple comparisons corrections, all significant comparisons survived.

\subsection{Microglial activation and tau pathology along the Alzheimer's clinical spectrum}

We next stratified subjects who had ${ }^{11} \mathrm{C}$-PBR28 PET along the Alzheimer's clinical spectrum, including amyloid-negative controls (normal aging, $\mathrm{n}=17$ ), amyloid-positive controls (early Alzheimer's pathophysiological change, $n=7$ ), amyloid-positive patients with $\mathrm{MCI}$ (the clinical prodrome of $\mathrm{AD}, \mathrm{n}=7$ ), and amyloid-positive patients with $\mathrm{AD}$ dementia (full clinical expression of the disease, $\mathrm{n}=16$ ). We found that ${ }^{11} \mathrm{C}$-PBR28 binding increased in a stepwise manner, particularly in neocortical ROIs (Fig 2A, Table 4). Thus, each disease stage showed greater ${ }^{11} \mathrm{C}$-PBR28 binding than an earlier disease stage, with temporal and parietal ROIs showing the largest differences.

We then stratified subjects who had ${ }^{18} \mathrm{~F}-\mathrm{MK}-6240$ PET along the Alzheimer's clinical spectrum, including amyloid-negative controls $(\mathrm{n}=12)$, amyloid-positive controls $(\mathrm{n}=7)$, amyloid-positive patients with $\mathrm{MCI}(\mathrm{n}=4)$, and amyloid-positive patients with $\mathrm{AD}$ dementia $(n=10)$. We found that ${ }^{18} \mathrm{~F}-\mathrm{MK}-6240$ binding increased along the $\mathrm{AD}$ spectrum; however, the pattern was different from that seen with ${ }^{11} \mathrm{C}-\mathrm{PBR} 28$ (Fig 2B, Table 5). The earliest Alzheimer's pathophysiological change was associated with increase in ${ }^{18} \mathrm{~F}-\mathrm{MK}$ 6240 binding in medial temporal cortex only, with increase in neocortical binding seen only in the $\mathrm{MCI}$ and $\mathrm{AD}$ groups.

Correlations between ${ }^{11} \mathrm{C}$-PBR28 binding and MMSE score showed that ${ }^{11} \mathrm{C}$-PBR28 binding was negatively associated with MMSE score in all ROIs except striatum ( $p$ 's $<0.01$ ) when all participants were combined. When we stratified participants based on 
Table 4

${ }^{11}$ C-PBR28 SUVR values (partial volume corrected) across the clinical Alzheimer's spectrum

\begin{tabular}{|c|c|c|c|c|c|c|c|c|c|c|}
\hline \multirow[t]{2}{*}{ Region } & \multicolumn{4}{|c|}{${ }^{11} \mathrm{C}-\mathrm{PBR} 28$ binding (SUVR) } & \multicolumn{6}{|c|}{ Groupwise comparison $^{\mathrm{a}}$} \\
\hline & $\begin{array}{l}\mathrm{A} \beta(-) \text { controls } \\
(\mathrm{n}=17)\end{array}$ & $\begin{array}{l}\mathrm{A} \beta(+) \text { controls } \\
(\mathrm{n}=7)\end{array}$ & $\begin{array}{l}\mathrm{A} \beta(+) \mathrm{MCI} \\
(\mathrm{n}=7)\end{array}$ & $\begin{array}{l}\mathrm{A} \beta(+) \mathrm{ADD} \\
(\mathrm{n}=16)\end{array}$ & $\begin{array}{l}A \beta(-) \text { controls vs. } \\
A \beta(+) \text { controls }\end{array}$ & $\begin{array}{l}\mathrm{A} \beta(-) \text { controls vs. } \\
\mathrm{A} \beta(+) \mathrm{MCI}\end{array}$ & $\begin{array}{l}A \beta(-) \text { controls vs. } \\
A \beta(+) A D D\end{array}$ & $\begin{array}{l}\mathrm{A} \beta(+) \text { controls vs. } \\
\mathrm{A} \beta(+) \mathrm{MCI}\end{array}$ & $\begin{array}{l}A \beta(+) \text { controls vs. } \\
A \beta(+) A D D\end{array}$ & $\begin{array}{l}\mathrm{A} \beta(+) \mathrm{MCI} \text { vs. } \\
\mathrm{A} \beta(+) \mathrm{ADD}\end{array}$ \\
\hline Prefrontal & $1.10 \pm 0.17$ & $1.21 \pm 0.07$ & $1.23 \pm 0.08$ & $1.29 \pm 0.08$ & $-1.9688(0.0554)$ & $-2.4681(0.0176)$ & $-4.5680(<0.0001)$ & $-0.4196(0.6769)$ & $-1.5601(0.1261)$ & $-1.0652(0.2927)$ \\
\hline Mid/inf temporal & $1.04 \pm 0.10$ & $1.13 \pm 0.06$ & $1.15 \pm 0.07$ & $1.28 \pm 0.13$ & $-2.0137(0.0503)$ & $-2.4234(0.0197)$ & $-6.6251(<0.0001)$ & $-0.3442(0.7324)$ & $-3.0967 /(0.0034)$ & $-2.6907(0.0101)$ \\
\hline Superior temporal & $1.09 \pm 0.13$ & $1.19 \pm 0.07$ & $1.19 \pm 0.07$ & $1.25 \pm 0.08$ & $-2.2802(0.0276)$ & $-2.1303(0.0389)$ & $-4.3732(<0.0001)$ & $-0.1260(0.9003)$ & $-1.1017(0.2767)$ & $-1.2503(0.2180)$ \\
\hline Medial temporal & $1.02 \pm 0.13$ & $1.15 \pm 0.16$ & $1.20 \pm 0.17$ & $1.27 \pm 0.19$ & $-1.7648(0.0847)$ & $-2.4803(0.0171)$ & $-4.4196(<0.0001)$ & $-0.6011(0.5509)$ & $-1.6481(0.1066)$ & $-0.9391(0.3529)$ \\
\hline Posterior cingulate & $1.09 \pm 0.12$ & $1.13 \pm 0.08$ & $1.21 \pm 0.13$ & $1.30 \pm 0.10$ & $-0.6557(0.5155)$ & $-2.3127(0.0256)$ & $-5.4455(<0.0001)$ & $-1.3922(0.1710)$ & $-3.5358(0.0010)$ & $-1.8937(0.0650)$ \\
\hline Superior parietal & $1.04 \pm 0.16$ & $1.16 \pm 0.11$ & $1.25 \pm 0.14$ & $1.30 \pm 0.11$ & $-2.0893(0.0426)$ & $-3.6251(0.0008)$ & $-5.7561(<0.0001)$ & $-1.2904(0.2038)$ & $-2.3538(0.0232)$ & $-0.8318(0.4101)$ \\
\hline Inferior parietal & $1.00 \pm 0.15$ & $1.11 \pm 0.08$ & $1.19 \pm 0.11$ & $1.27 \pm 0.11$ & $-1.9123(0.0625)$ & $-3.4927(0.0011)$ & $-6.4202(<0.0001)$ & $-1.3278(0.1913)$ & $-3.0397(0.0040)$ & $-1.4735(0.1479)$ \\
\hline Striatum & $0.82 \pm 0.13$ & $0.81 \pm 0.07$ & $0.83 \pm 0.08$ & $0.86 \pm 0.08$ & $-0.1426(0.8873)$ & $-0.2398(0.8117)$ & $-1.2434(0.2205)$ & $-0.3212(0.7496)$ & $-1.0970(0.2787)$ & $-0.7181(0.4766)$ \\
\hline
\end{tabular}

Key: ADD, Alzheimer's disease dementia; MCI, mild cognitive impairment; SUVR, standardized uptake value ratio

a Results given as $\mathrm{t}$ statistics, with $p$ values in parentheses.

Table 5

${ }^{18} \mathrm{~F}-\mathrm{MK}-6240$ SUVR values (partial volume-corrected) across the clinical Alzheimer's spectrum

\begin{tabular}{|c|c|c|c|c|c|c|c|c|c|c|}
\hline \multirow[t]{2}{*}{ Region } & \multicolumn{4}{|c|}{${ }^{18} \mathrm{~F}-\mathrm{MK}-6240$ binding (SUVR) } & \multicolumn{6}{|c|}{ Groupwise comparison $^{\mathrm{a}}$} \\
\hline & $\begin{array}{l}\mathrm{A} \beta(-) \text { controls } \\
(\mathrm{n}=12)\end{array}$ & $\begin{array}{l}\mathrm{A} \beta(+) \text { controls } \\
(\mathrm{n}=7)\end{array}$ & $\begin{array}{l}\mathrm{A} \beta(+) \mathrm{MCI} \\
(\mathrm{n}=4)\end{array}$ & $\begin{array}{l}\mathrm{A} \beta(+) \mathrm{ADD} \\
(\mathrm{n}=10)\end{array}$ & $\begin{array}{l}\mathrm{A} \beta(-) \text { controls vs. } \\
\mathrm{A} \beta(+) \text { controls }\end{array}$ & $\begin{array}{l}A \beta(-) \text { controls vs. } \\
A \beta(+) M C I\end{array}$ & $\begin{array}{l}A \beta(-) \text { controls vs. } \\
A \beta(+) A D D\end{array}$ & $\begin{array}{l}\mathrm{A} \beta(+) \text { controls vs. } \\
\mathrm{A} \beta(+) \mathrm{MCI}\end{array}$ & $\begin{array}{l}A \beta(+) \text { controls vs. } \\
A \beta(+) A D D\end{array}$ & $\begin{array}{l}\mathrm{A} \beta(+) \mathrm{MCl} \text { vs. } \\
\mathrm{A} \beta(+) \mathrm{ADD}\end{array}$ \\
\hline Prefrontal & $1.13 \pm 0.14$ & $1.29 \pm 0.24$ & $2.22 \pm 0.95$ & $4.43 \pm 2.54$ & $-0.2401(0.8120)$ & $-1.2986(0.2043)$ & $-5.2919(<0.0001)$ & $-1.0140(0.3190)$ & $-4.3662(0.0001)$ & $-2.5627(0.0158)$ \\
\hline Mid/inf temporal & $1.25 \pm 0.14$ & $1.76 \pm 0.71$ & $2.91 \pm 1.07$ & $4.88 \pm 2.06$ & $-0.8609(0.3963)$ & $-2.3011(0.0288)$ & $-6.7992(<0.0001)$ & $-1.4663(0.1533)$ & $-5.0766(<0.0001)$ & $-2.6753(0.0121)$ \\
\hline Superior temporal & $1.15 \pm 0.16$ & $1.35 \pm 0.27$ & $2.59 \pm 1.05$ & $3.72 \pm 1.77$ & $-0.3977(0.6938)$ & $-2.3791(0.0242)$ & $-5.7029(<0.0001)$ & $-1.8897(0.0688)$ & $-4.5712(<0.0001)$ & $-1.8057(0.0814)$ \\
\hline Medial temporal & $1.11 \pm 0.31$ & $2.04 \pm 1.17$ & $3.58 \pm 0.17$ & $3.98 \pm 1.30$ & $-2.1327(0.0415)$ & $-4.6544(<0.0001)$ & $-7.3027(<0.0001)$ & $-2.6691(0.0123)$ & $-4.2868(0.0002)$ & $-0.7431(0.4634)$ \\
\hline Posterior cingulate & $1.00 \pm 0.12$ & $1.14 \pm 0.12$ & $2.99 \pm 1.96$ & $5.44 \pm 2.56$ & $-0.1855(0.85)$ & $-2.2030(0.0357)$ & $-6.6353(<0.0001)$ & $-1.8885(0.0690)$ & $-5.5861(<0.0001)$ & $-2.6523(0.0128)$ \\
\hline Superior parietal & $1.19 \pm 0.13$ & $1.43 \pm 0.25$ & $4.94 \pm 4.14$ & $6.21 \pm 3.54$ & $-0.2139(0.8322)$ & $-2.7228(0.0108)$ & $-4.9176(<0.0001)$ & $-2.3458(0.0260)$ & $-4.0663(0.0003)$ & $-0.9019(0.3745)$ \\
\hline Inferior parietal & $1.16 \pm 0.15$ & $1.45 \pm 0.45$ & $4.36 \pm 3.20$ & $6.15 \pm 3.03$ & $-0.3085(0.7599)$ & $-2.7770(0.0095)$ & $-5.8532(<0.0001)$ & $-2.3239(0.0273)$ & $-4.7878(<0.0001)$ & $-1.5262(0.1378)$ \\
\hline Striatum & $0.71 \pm 0.15$ & $0.88 \pm 0.12$ & $0.90 \pm 0.23$ & $1.20 \pm 0.35$ & $-1.4756(0.1508)$ & $-1.3644(0.1829)$ & $-4.8862(<0.0001)$ & $-0.1371(0.8919)$ & $-2.8213(0.0085)$ & $-2.2049(0.0355)$ \\
\hline
\end{tabular}

Key: ADD, Alzheimer's disease dementia; MCI, mild cognitive impairment; SUVR, standardized uptake value ratio.

Key: ADD, Alzheimer's disease dementia; $M$ Res, mild cognitive
${ }^{2}$ Results given as t statistics, with $p$ values in parentheses. 
amyloid status, this negative correlation remained for amyloidpositive subjects in each ROI except medial temporal cortex ( $p$ 's < 0.05) (Fig 3A, Supplementary Fig 1). ${ }^{11}$ C-PBR28 binding did not correlate with MMSE score when only amyloid-negative participants were included.

Similar to ${ }^{11} \mathrm{C}$-PBR28, when all subjects who had ${ }^{18} \mathrm{~F}-\mathrm{MK}-6240$ PET were included $(n=43)$, binding negatively correlated with MMSE score in all ROIs, including striatum ( $p$ 's $<0.01)$. Negative correlations were seen among amyloid-positive subjects in all ROIs ( $p$ 's $<0.05)$ and no correlations were seen in amyloid-negative subjects when considered separately (Fig 3B, Supplementary Fig 2). Correlation analyses using ${ }^{11}$ C-PBR28 and ${ }^{18}$ F-MK-6240 data uncorrected for partial volume effects followed a similar pattern (Supplementary Fig 3 and 4).

\subsection{Correlation between microglial activation and tau deposition}

For subjects with both ${ }^{18} \mathrm{~F}-\mathrm{MK}-6240$ PET and ${ }^{11} \mathrm{C}$-PBR28 PET performed ( $\mathrm{n}=42$ ), we performed a correlation between these radioligands to look for regional relationships between TSPO and tau. Using the partial volume-corrected SUVR data, we found a significant positive correlation between ${ }^{18} \mathrm{~F}-\mathrm{MK}-6240$ and ${ }^{11} \mathrm{C}$ PBR28 binding in every region except the striatum ( $p$ 's $<0.005$ ). Results from correlation analysis using imaging data uncorrected for partial volume effects followed a similar pattern (Supplementary Table 3).

\section{Discussion}

We demonstrated that amyloid positivity and cognitive impairment are independently associated with ${ }^{11} \mathrm{C}$-PBR2 28 binding. These results suggest that increased microglial activation co-occurs with amyloid plaque deposition in the absence of cognitive impairment and with cognitive impairment in the absence of amyloidosis. Because cognitive impairment is the clinical result of neurodegeneration, our results argue that microglial activation could be both a specific response to amyloid plaque deposition in early stages of $\mathrm{AD}$ and a nonspecific response to neurodegeneration in both Alzheimer's and non-Alzheimer's dementias. By contrast, amyloid and impairment interact in their association with ${ }^{18} \mathrm{~F}-\mathrm{MK}$ 6240 binding, such that binding is synergistically increased in amyloid-positive patients but not increased in amyloid-negative patients. These results suggest that tau pathology is most often seen when both amyloid and cognitive impairment are both already present. That ${ }^{11} \mathrm{C}-\mathrm{PBR} 28$ and ${ }^{18} \mathrm{~F}-\mathrm{MK}-6240$ binding negatively correlate with MMSE score among amyloid-positive subjects suggests a unidirectional increase in both microglial activation and tau pathology throughout the progression of AD.

We also found that ${ }^{11} \mathrm{C}-\mathrm{PBR} 28$ and ${ }^{18} \mathrm{~F}-\mathrm{MK}-6240$ showed different patterns of binding along the $\mathrm{AD}$ clinical spectrum. ${ }^{11} \mathrm{C}$ PBR28 binding increased in a stepwise pattern in temporoparietal neocortex, starting in amyloid-positive controls. By contrast, ${ }^{18} \mathrm{~F}$ MK-6240 binding was increased in amyloid-positive controls only in medial temporal cortex, with neocortical increases only seen in $\mathrm{MCI}$ and $\mathrm{AD}$ patients. These results seem to agree with earlier observations with ${ }^{18} \mathrm{~F}-\mathrm{AV}-1451$ that showed amyloid-positive controls were more likely than amyloid-negative controls to have high tau PET signal, particularly in medial temporal regions (Lowe et al., 2017). Notably, in our study, we found an interaction between amyloid positivity and cognitive impairment on ${ }^{18} \mathrm{~F}-\mathrm{MK}-6240$ binding in the medial temporal cortex $(p<0.01)$, suggesting that even in this region increased tau pathology is related to antecedent amyloid deposition. These results suggest a possible temporal order of Alzheimer's pathophysiology beginning with deposition of amyloid plaque, subsequent microglial activation in the neocortex, and increased tau pathology in the medial temporal cortex. These events are then followed by the appearance of increased tau pathology in neocortex and concurrent onset of memory impairment. Alternatively, it is possible that neuroinflammation and tau deposition occur at the same time, as ${ }^{18} \mathrm{~F}-\mathrm{MK} 6240$ may be less sensitive than ${ }^{11} \mathrm{C}$-PBR28 in detecting small changes in pathology. In either case, these cross-sectional findings require confirmation in a longitudinal study as conclusions about temporal order cannot be made in a cross-sectional study.

That amyloid positivity and memory impairment are independently associated with increased ${ }^{11} \mathrm{C}$-PBR28 binding is consistent with distinct roles of microglial activation at different time points: an early response to amyloidosis and a later response to neurodegeneration, the latter not necessarily specific to Alzheimer's pathogenesis (Sierra et al., 2013). Increased TSPO binding in amyloid-positive controls has been previously reported (Hamelin et al., 2016). Downstream response of microglia to neurodegeneration has been demonstrated by their activation in the presence of axonal injury (Tanaka et al., 2009), and would explain increased TSPO binding in a variety of neurodegenerative diseases (Schain and Kreisl, 2017). However, imaging studies alone cannot determine
A

Inferior parietal lobule

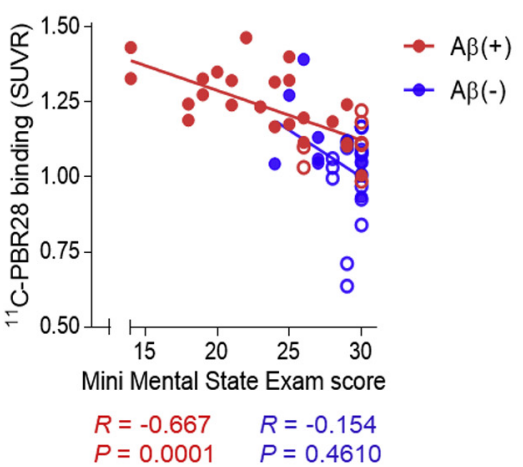

B

Inferior parietal lobule

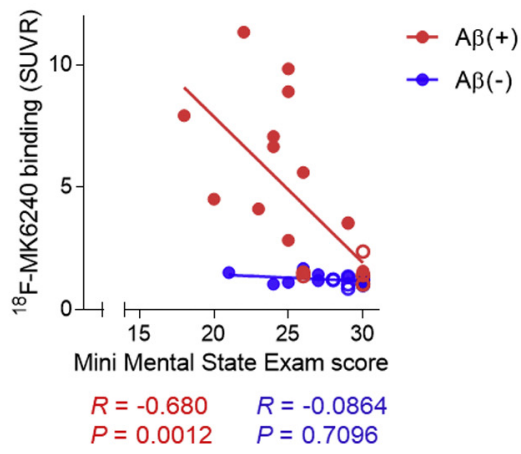

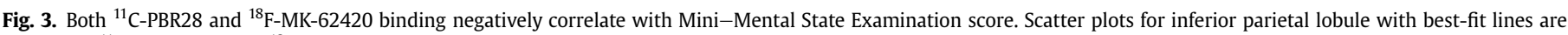

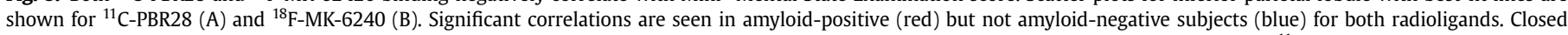

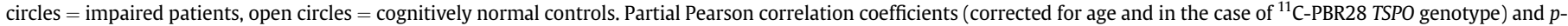

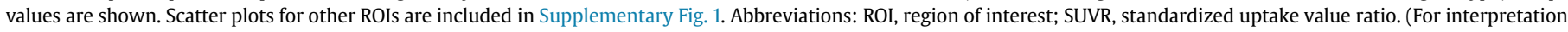
of the references to color in this figure legend, the reader is referred to the Web version of this article.) 
the functional role of microglia in AD. Whether microglial activation plays a protective or pathogenic role in the preclinical stage remains unclear, as both phagocytic behavior and release of neurotoxic cytokines have been observed by microglia in response to $\beta$-amyloid species in vitro (D’Andrea et al., 2004; Maezawa et al., 2011). Although some investigators have posited a bimodal relationship between TSPO and AD progression (Calsolaro and Edison, 2016; Fan et al., 2017; Hamelin et al., 2016), the independent associations of ${ }^{11} \mathrm{C}$-PBR28 binding with amyloid positivity and cognitive impairment, and apparent linear increase in ${ }^{11} \mathrm{C}$-PBR28 binding along the disease spectrum, suggest a unimodal increase in overall microglial activation throughout the Alzheimer's process.

The underlying pathologies of the included amyloid-negative subjects with cognitive impairment are not known, but no participant had cardinal symptoms of dementia with Lewy bodes, frontotemporal dementia, progressive supranuclear palsy, corticobasal syndrome, or vascular dementia. Our amyloid-negative patients, on average, had smaller hippocampal volumes than our amyloidnegative controls, suggesting these patients in general had an Alzheimer's-like pattern of neurodegeneration. Hippocampal sclerosis and argyrophilic grain disease are $2 \mathrm{AD}$ mimics potentially represented among patients lacking Alzheimer's pathophysiology (Jicha et al., 2006). Because cognitive impairment was associated with increased ${ }^{11} \mathrm{C}$-PBR28 binding in the absence of amyloid pathophysiology, TSPO PET could potentially be used as a disease marker in non-Alzheimer amnestic disorders for which no in vivo molecular biomarkers yet exist.

To our knowledge, this is the first study to use ${ }^{11} \mathrm{C}-\mathrm{PBR} 28$ to measure microglial activation and ${ }^{18} \mathrm{~F}-\mathrm{MK}-6240$ to measure tau pathology in the same participants. Prior studies used ${ }^{11} \mathrm{C}-(R)-\mathrm{PK} 11195$ to measure TSPO binding (Parbo et al., 2018) and/or ${ }^{18} \mathrm{~F}-\mathrm{AV}-1451$ to measure tau binding (Dani et al., 2018). ${ }^{11} \mathrm{C}-\mathrm{PBR} 28$ has greater specific-to-nonspecific binding than ${ }^{11} \mathrm{C}-(R)-\mathrm{PK} 11195$ (Fujita et al., 2017; Kreisl et al., 2010); has been validated in AD subjects using a 30 minutes imaging window (Lyoo et al., 2015); has demonstrated longitudinal increases in AD (Kreisl et al., 2016); and co-localizes to neurodegeneration in vivo (Kreisl et al., 2017b) and microglial activation ex vivo (Kreisl et al., 2017a). Based on our prior study (Lyoo et al., 2015), SUVR measurement of ${ }^{11}$ C-PBR28 binding results in greater statistical power than kinetic modeling using the arterial input function in detecting differences between $A D$ patients and controls. Therefore, the SUVR approach with ${ }^{11} \mathrm{C}$-PBR28 may be preferred over full quantification for detecting small differences in ${ }^{11}$ C-PBR28 binding, such as that seen between amyloid-negative and amyloid-positive control groups. We recently demonstrated that using supervised clustering analysis also resulted in improved ability to detect increased ${ }^{11} \mathrm{C}$-PBR28 binding in patients with AD compared with kinetic modeling with the arterial input function (Fregonara et al., 2019). Although using SUVR has not been directly compared with using supervised clustering analysis, either approach appears to be a suitable alternative to arterial sampling, at least in cross-sectional studies. The SUVR approach has the additional advantage of shorter imaging times (and therefore reduced participant burden).

${ }^{18} \mathrm{~F}-\mathrm{MK}-6240$ appears to have less off-target binding in choroid plexus and basal ganglia than ${ }^{18} \mathrm{~F}-\mathrm{AV}-1451$ (Betthauser et al., 2019). Moreover, the 2 studies using ${ }^{18} \mathrm{~F}-\mathrm{AV}-1451$ showed somewhat discordant results, with one showing a correlation between these 2 radioligands (Dani et al., 2018) and the other finding no correlation (Parbo et al., 2018). Interestingly, our results are more in line with that of Dani et al., which similarly used ${ }^{11} \mathrm{C}$-PBR28 for TSPO imaging. Using ${ }^{11} \mathrm{C}$-PBR28 and ${ }^{18} \mathrm{~F}-\mathrm{MK}-6240$ together may have increased the sensitivity of detecting increased TSPO and tau pathology in our study.

Correction for partial volume effects is an important consideration in AD studies-as atrophy of the cerebral cortex exacerbates the partial volume effects from CSF inherent in PET imaging-and may be a more accurate representation of true radioligand activity (Su et al., 2015). Although corrected data resulted in larger effect sizes, the direction of the relationships between radioligand binding and the comparative outcome variable remained the same regardless of whether partial volume correction was applied (see Supplementary Information). In addition, that we still found correlations between ${ }^{11} \mathrm{C}$-PBR28 and ${ }^{18} \mathrm{~F}-\mathrm{MK}-6240$ and MMSE score in amyloid-positive subjects in partial volume-uncorrected image data argues that our results were not simply due to over-estimation caused by partial volume correction.

Our conclusions are somewhat limited by our sample size. Although we obtained PET imaging in 58 participants overall, only 10 amyloid-negative patients and 7 amyloid-positive controls had ${ }^{11}$ C-PBR28 PET imaging. In addition, the smaller number of participants in these 2 groups reflects the lower likelihood of amyloidnegativity in amnestic patients with $\mathrm{AD}$ patterns of neurodegeneration and of amyloid-positivity in controls, particularly given the relatively young ages included $(69.2 \pm 4.3$ years for controls overall). While enrolling older participants is expected to increase the prevalence of amyloid-positivity in controls (Chetelat et al., 2013), this approach would also increase the prevalence of non-Alzheimer co-pathology in the amyloid-positive patient group. The young age of amyloid-positive patient group $(65.8 \pm 8.4$ years $)$ reduces the likelihood that concomitant neurodegenerative disorders influenced our results. That ${ }^{11} \mathrm{C}$-PBR28 binding is influenced by TSPO genotype introduces an element of variability in the analysis, and is a shortcoming of this radioligand. However, excluding low affinity binders and including TSPO genotype in the statistical analysis, as performed in this study, has been shown to overcome the confounding effects of this nuisance variable (Kreisl et al., 2013a, b). Another limitation of our study is that only a subset of participants underwent ${ }^{18} \mathrm{~F}-\mathrm{MK}-6240$ PET. However, the large dynamic range of this radioligand and large effects sizes seen in ours and prior studies (Betthauser et al., 2019; Lohith et al., 2019; Pascoal et al., 2018) argue that even modestly sized studies have sufficient power to see group differences in ${ }^{18} \mathrm{~F}-\mathrm{MK}-6240$ binding.

In conclusion, amyloid positivity and memory impairment are independently related to ${ }^{11} \mathrm{C}$-PBR28 binding. By contrast, amyloid and impairment interact in their relationship with ${ }^{18} \mathrm{~F}-\mathrm{MK}-6240$ binding.

\section{Disclosure}

Dr. Kreisl is a consultant for Cerveau Technologies. However, Cerveau was not involved in the design or execution of this study or in the interpretation of the results.

\section{Acknowledgements}

${ }^{18} \mathrm{~F}$-Florbetaben was supplied by Life Molecular Imaging. ${ }^{18} \mathrm{~F}$ MK-6240 was supplied by Cerveau Technologies. TSPO genotyping was performed by Regina Santella, PhD, and the Columbia University Biomarkers Shared Resource. The authors wish to acknowledge the faculty and staff at the Irving Institute Clinical Research Resource, the MRI Center, and the David A. Gardner PET Imaging Research Center at the Columbia University Irving Medical Center for their contributions to this work.

This study was supported by National Institutes of Health grants K23AG052633 (WCK), R01AG026158(YS) and R56AG034189 (AMB). This study was supported by Columbia University's CTSA grant (UL1 TR000040), and the Columbia University Alzheimer's Disease Research Center(P50AG008702). Data collection and sharing for this project was supported by the Washington Heights-Inwood Columbia Aging Project (WHICAP, P01AG07232, R01AG037212, 
RF1AG054023). This manuscript has been reviewed by WHICAP investigators for scientific content and consistency of data interpretation with previous WHICAP Study publications. The authors acknowledge the WHICAP study participants and the WHICAP research and support staff for their contributions to this study.

\section{Appendix A. Supplementary data}

Supplementary data to this article can be found online at https:// doi.org/10.1016/j.neurobiolaging.2019.09.019.

\section{References}

Albert, M.S., DeKosky, S.T., Dickson, D., Dubois, B., Feldman, H.H., Fox, N.C., Gamst, A., Holtzman, D.M., Jagust, W.J., Petersen, R.C., Snyder, P.J., Carillo, M.C., Thies, B., Phelps, C.H., 2011. The diagnosis of mild cognitive impairment due to Alzheimer's disease: recommendations from the National Institute on Aging-Alzheimer's Association workgroups on diagnostic guidelines for Alzheimer's disease. Alzheimers Dement. 7, 270-279.

Betthauser, T.J., Cody, K.A., Zammit, M.D., Murali, D., Converse, A.K., Barnhart, T.E., Stone, C.K., Rowley, H.A., Johnson, S.C., Christian, B.T., 2019. In vivo characterization and quantification of neurofibrillary tau PET radioligand (18)F-MK-6240 in humans from Alzheimer disease dementia to young controls. J. Nucl. Med. 60, 93-99.

Briard, E., Zoghbi, S.S., Imaizumi, M., Gourley, J.P., Shetty, H.U., Hong, J., Cropley, V., Fujita, M., Innis, R.B., Pike, V.W., 2008. Synthesis and evaluation in monkey of two sensitive 11C-labeled aryloxyanilide ligands for imaging brain peripheral benzodiazepine receptors in vivo. J. Med. Chem. 51, 17-30.

Bullich, S., Seibyl, J., Catafau, A.M., Jovalekic, A., Koglin, N., Barthel, H., Sabri, O., De Santi, S., 2017. Optimized classification of (18)F-Florbetaben PET scans as positive and negative using an SUVR quantitative approach and comparison to visual assessment. Neuroimage Clin. 15, 325-332.

Cagnin, A., Rossor, M., Sampson, E.L., Mackinnon, T., Banati, R.B., 2004. In vivo detection of microglial activation in frontotemporal dementia. Ann. Neurol. 56, 894-897.

Calsolaro, V., Edison, P., 2016. Neuroinflammation in Alzheimer's disease: current evidence and future directions. Alzheimers Dement. 12, 719-732.

Chetelat, G., La Joie, R., Villain, N., Perrotin, A., de La Sayette, V., Eustache, F., Vandenberghe, R., 2013. Amyloid imaging in cognitively normal individuals, atrisk populations and preclinical Alzheimer's disease. Neuroimage Clin. 2, 356-365.

Colonna, M., Wang, Y., 2016. TREM2 variants: new keys to decipher Alzheimer disease pathogenesis. Nat. Rev. Neurosci. 17, 201-207.

Corcia, P., Tauber, C., Vercoullie, J., Arlicot, N., Prunier, C., Praline, J., Nicolas, G., Venel, Y., Hommet, C., Baulieu, J.L., Cottier, J.P., Roussel, C., Kassiou, M., Guilloteau, D., Ribeiro, M.J., 2012. Molecular imaging of microglial activation in amyotrophic lateral sclerosis. PLoS One 7, e52941.

D’Andrea, M.R., Cole, G.M., Ard, M.D., 2004. The microglial phagocytic role with specific plaque types in the Alzheimer disease brain. Neurobiol. Aging 25, 675-683.

Dani, M., Wood, M., Mizoguchi, R., Fan, Z., Walker, Z., Morgan, R., Hinz, R., Biju, M., Kuruvilla, T., Brooks, D.J., Edison, P., 2018. Microglial activation correlates in vivo with both tau and amyloid in Alzheimer's disease. Brain 141, 2740-2754.

Edison, P., Archer, H.A., Gerhard, A., Hinz, R., Pavese, N., Turkheimer, F.E., Hammers, A., Tai, Y.F., Fox, N., Kennedy, A., Rossor, M., Brooks, D.J., 2008. Microglia, amyloid, and cognition in Alzheimer's disease: an [11C](R)PK11195PET and [11C]PIB-PET study. Neurobiol. Dis. 32, 412-419.

Fan, Z., Brooks, D.J., Okello, A., Edison, P., 2017. An early and late peak in microglial activation in Alzheimer's disease trajectory. Brain 140, 792-803.

Folstein, M.F., Robins, L.N., Helzer, J.E., 1983. The mini-mental state examination. Arch. Gen. Psychiatry 40, 812.

Fregonara, P.Z., Kreisl, W.C., Innis, R.B., Lyoo, C.H., 2019. Automatic extraction of a reference region for the noninvasive quantification of translocator protein in brain using 11C-PBR28. J. Nucl. Med. 60, 978-984.

Fujita, M., Kobayashi, M., Ikawa, M., Gunn, R.N., Rabiner, E.A., Owen, D.R., Zoghbi, S.S., Haskall, M.B., Telu, S., Pike, V.W., Innis, R.B., 2017. Comparison of four (11)C-labeled PET ligands to quantify translocator protein $18 \mathrm{kDa}$ (TSPO) in human brain: (R)-PK11195, PBR28, DPA-713, and ER176-based on recent publications that measured specific-to-non-displaceable ratios. EJNMMI Res. 7, 84.

Gerhard, A., Pavese, N., Hotton, G., Turkheimer, F., Es, M., Hammers, A., Eggert, K., Oertel, W., Banati, R.B., Brooks, D.J., 2006. In vivo imaging of microglial activation with $[11 \mathrm{C}](\mathrm{R})-\mathrm{PK} 11195$ PET in idiopathic Parkinson's disease. Neurobiol. Dis. 21, 404-412.

Hamelin, L., Lagarde, J., Dorothee, G., Leroy, C., Labit, M., Comley, R., de Souza, L.C., Corne, H., Dauphinot, L., Bertoux, M., Dubois, B., Gervais, P., Colliot, O., Potier, M.C., Bottlaender, M., Sarazin, M., the Clinical IMABio3 team, 2016. Early and protective microglial activation in Alzheimer's disease: a prospective study using 18F-DPA-714 PET imaging. Brain 139 (Pt 4), 1252-1264.

Hostetler, E.D., Walji, A.M., Zeng, Z., Miller, P., Bennacef, I., Salinas, C., Connolly, B., Gantert, L., Haley, H., Holahan, M., Purcell, M., Riffel, K., Lohith, T.G., Coleman, P., Soriano, A., Ogawa, A., Xu, S., Zhang, X., Joshi, E., Della Rocca, J., Hesk, D.,
Schenk, D.J., Evelhoch, J.L., 2016. Preclinical characterization of 18F-MK-6240, a promising PET tracer for in vivo quantification of human neurofibrillary tangles. J. Nucl. Med. 57, 1599-1606.

Hughes, C.P., Berg, L., Danziger, W., Coben, L.A., Martin, R.L., 1982. A new clinical scale for the staging of dementia. Br. J. Psychiatry 140, 566-572.

Jack Jr., C.R., Knopman, D.S., Jagust, W.J., Petersen, R.C., Weiner, M.W., Aisen, P.S., Shaw, L.M., Vemuri, P., Wiste, H.J., Weigand, S.D., Lesnick, T.G., Pankratz, V.S., Donohue, M.C., Trojanowski, J.Q., 2013. Tracking pathophysiological processes in Alzheimer's disease: an updated hypothetical model of dynamic biomarkers. Lancet Neurol. 12, 207-216.

Jack Jr., C.R., Wiste, H.J., Schwarz, C.G., Lowe, V.J., Senjem, M.L., Vemuri, P. Weigand, S.D., Therneau, T.M., Knopman, D.S., Gunter, J.L., Jones, D.T., GraffRadford, J., Kantarci, K., Roberts, R.O., Mielke, M.M., Machulda, M.M., Petersen, R.C., 2018. Longitudinal tau PET in ageing and Alzheimer's disease. Brain 141, 1517-1528.

Janelidze, S., Mattsson, N., Stomrud, E., Lindberg, O., Palmqvist, S., Zetterberg, H. Blennow, K. Hansson, O., 2018. CSF biomarkers of neuroinflammation and cerebrovascular dysfunction in early Alzheimer disease. Neurology 91, e867-e877.

Jicha, G.A., Parisi, J.E., Dickson, D.W., Johnson, K., Chia, R., Ivnik, R.J., Tangalos, E.G. Boeve, B.F., Knopman, D.S., Braak, H., Petersen, R.C., 2006. Neuropathologic outcome of mild cognitive impairment following progression to clinical dementia. Arch. Neurol. 63, 674-681.

Kreisl, W.C., Fujita, M., Fujimura, Y., Kimura, N., Jenko, K.J., Kannan, P., Hong, J., Morse, C.L., Zoghbi, S.S., Gladding, R.L., Jacobson, S., Oh, U., Pike, V.W., Innis, R.B., 2010. Comparison of [(11)C]-(R)-PK 11195 and [(11)C]PBR28, two radioligands for translocator protein $(18 \mathrm{kDa})$ in human and monkey: implications for positron emission tomographic imaging of this inflammation biomarker. Neuroimage 49, 2924-2932.

Kreisl, W.C., Jenko, K.J., Hines, C.S., Lyoo, C.H., Corona, W., Morse, C.L., Zoghbi, S.S. Hyde, T., Kleinman, J.E., Pike, V.W., McMahon, F.J., Innis, R.B., 2013a. A genetic polymorphism for translocator protein $18 \mathrm{kDa}$ affects both in vitro and in vivo radioligand binding in human brain to this putative biomarker of neuroinflammation. J. Cereb. Blood Flow Metab. 33, 53-58.

Kreisl, W.C., Lawrence, R., Page, E., Teich, A.F., Subramaniam, D.S., Innis, R.B. Turner, R.S., 2017a. (11)C-PBR28 PET detects translocator protein in a patient with astrocytoma and Alzheimer disease. Neurology 88, 1001-1004.

Kreisl, W.C., Lyoo, C.H., Liow, J.S., Snow, J., Page, E., Jenko, K.J., Morse, C.L. Zoghbi, S.S., Pike, V.W., Turner, R.S., Innis, R.B., 2017b. Distinct patterns of increased translocator protein in posterior cortical atrophy and amnestic Alzheimer's disease. Neurobiol. Aging 51, 132-140.

Kreisl, W.C., Lyoo, C.H., Liow, J.S., Wei, M., Snow, J., Page, E., Jenko, K.J., Morse, C.L. Zoghbi, S.S., Pike, V.W., Turner, R.S., Innis, R.B., 2016. (11)C-PBR28 binding to translocator protein increases with progression of Alzheimer's disease. Neurobiol. Aging 44, 53-61.

Kreisl, W.C., Lyoo, C.H., McGwier, M., Snow, J., Jenko, K.J., Kimura, N., Corona, W. Morse, C.L., Zoghbi, S.S., Pike, V.W., McMahon, F.J., Turner, R.S., Innis, R.B., the Biomarkers Consortium PET Radioligand Project Team, 2013b. In vivo radioligand binding to translocator protein correlates with severity of Alzheimer's disease. Brain 136 (Pt 7), 2228-2238.

Landau, S.M., Horng, A., Fero, A., Jagust, W.J., For the Alzheimer's Disease Neuroimaging Initiative, 2016. Amyloid negativity in patients with clinically diagnosed Alzheimer disease and MCI. Neurology 86, 1377-1385.

Lohith, T.G., Bennacef, I., Vandenberghe, R., Vandenbulcke, M., Salinas, C.A. Declercq, R., Reynders, T., Telan-Choing, N.F., Riffel, K., Celen, S., Serdons, K. Bormans, G., Tsai, K., Walji, A., Hostetler, E.D., Evelhoch, J.L., Van Laere, K. Forman, M., Stoch, A., Sur, C., Struyk, A., 2019. Brain imaging of Alzheimer dementia patients and elderly controls with (18)F-MK-6240, a PET tracer targeting neurofibrillary tangles. J. Nucl. Med. 60, 107-114.

Lowe, V.J., Wiste, H.J., Senjem, M.L., Weigand, S.D., Therneau, T.M., Boeve, B.F., Josephs, K.A., Fang, P., Pandey, M.K., Murray, M.E., Kantarci, K., Jones, D.T., Vemuri, P. Graff-Radford, J., Schwarz, C.G., Machulda, M.M., Mielke, M.M., Roberts, R.O. Knopman, D.S., Petersen, R.C., Jack Jr, C.R., 2017. Widespread brain tau and its association with ageing, Braak stage and Alzheimer's dementia. Brain 141, 271-287.

Lyoo, C.H., Ikawa, M., Liow, J.S., Zoghbi, S.S., Morse, C.L., Pike, V.W., Fujita, M., Innis, R.B., Kreisl, W.C., 2015. Cerebellum can serve as a pseudo-reference region in Alzheimer disease to detect neuroinflammation measured with PET radioligand binding to translocator protein. J. Nucl. Med. 56, 701-706.

Maezawa, I., Zimin, P.I., Wulff, H., Jin, L.W., 2011. Amyloid-beta protein oligomer at low nanomolar concentrations activates microglia and induces microglial neurotoxicity. J. Biol. Chem. 286, 3693-3706.

Marlatt, M.W., Bauer, J., Aronica, E., van Haastert, E.S., Hoozemans, J.J.M., Joels, M. Lucassen, P.J., 2014. Proliferation in the Alzheimer hippocampus is due to microglia, not astroglia, and occurs at sites of amyloid deposition. Neural Plast. 2014, 693851.

McKhann, G.M., Knopman, D.S., Chertkow, H., Hyman, B.T., Jack Jr, C.R., Kawas, C.H., Klunk, W.E., Koroshetz, W.J., Manly, J.J., Mayeux, R. Mohs, R.C., Morris, J.C., Rossor, M.N., Scheltens, P., Carillo, M.C., Thies, B., Weintraub, S., Phelps, C.H. 2011. The diagnosis of dementia due to Alzheimer's disease: recommendations from the National Institute on Aging-Alzheimer's Association workgroups on diagnostic guidelines for Alzheimer's disease. Alzheimers Dement. 7, 263-269.

Owen, D.R., Yeo, A.J., Gunn, R.N., Song, K., Wadsworth, G., Lewis, A., Rhodes, C., Pulford, D.J., Bennacef, I., Parker, C.A., St. Jean, P.L., Cardon, L.R., Mooser, V.E. Matthews, P.M., Rabiner, E.A., Rubio, J.P., 2012. An 18-kDa translocator protein (TSPO) polymorphism explains differences in binding affinity of the PET radioligand PBR28. J. Cereb. Blood Flow Metab. 32, 1-5. 
Parbo, P., Ismail, R., Sommerauer, M., Stokholm, M.G., Hansen, A.K., Hansen, K.V., Amidi, A., Schaldemose, J.L., Gottrup, H., Braendgaard, H., Eskildsen, S.F., Borghammer, P., Hinz, R., Aanerud, J., Brooks, D.J., 2018. Does inflammation precede tau aggregation in early Alzheimer's disease? A PET study. Neurobiol. Dis. 117, $211-216$

Pascoal, T.A., Shin, M., Kang, M.S., Chamoun, M., Chartrand, D., Mathotaarachchi, S., Bennacef, I., Therriault, J., Ng, K.P., Hopewell, R., Bouhachi, R., Hsiao, H.H., Benedet, A.L., Soucy, J.P., Massarweh, G., Gauthier, S., Rosa-Neto, P., 2018. In vivo quantification of neurofibrillary tangles with [(18)F]MK-6240. Alzheimers Res. Ther. 10, 74 .

Petersen, R.C., Aisen, P., Boeve, B.F., Geda, Y.E., Ivnik, R.J., Knopman, D.S., Mielke, M., Pankratz, V.S., Roberts, R., Rocca, W.A., Weigand, S., Weiner, M., Wiste, H., Jack Jr, C.R., 2013. Mild cognitive impairment due to Alzheimer disease in the community. Ann. Neurol. 74, 199-208.

Pike, K.E., Ellis, K.A., Villemagne, V.L., Good, N., Chetelat, G., Ames, D., Szoeke, C., Laws, S.M., Verdile, G., Martins, R.N., Masters, C.L., Rowe, C.C., 2011. Cognition and beta-amyloid in preclinical Alzheimer's disease: data from the AIBL study. Neuropsychologia 49, 2384-2390.

Ruff, R.M., Light, R.H., Guayhagen, M., 1989. Selective reminding tests: a normative study of verbal learning in adults. J. Clin. Exp. Neuropsychol. 11, $539-550$.
Schain, M., Kreisl, W.C., 2017. Neuroinflammation in neurodegenerative disorders-a Review. Curr. Neurol. Neurosci. Rep. 17, 25.

Schultz, S.A., Gordon, B.A., Mishra, S., Su, Y., Perrin, R.J. Cairns, N.J., Morris, J.C., Ances, B.M., Benzinger, T.L.S., 2018. Widespread distribution of tauopathy in preclinical Alzheimer's disease. Neurobiol. Aging 72, 177-185.

Sierra, A., Abiega, O., Shahraz, A., Neumann, H., 2013. Janus-faced microglia: beneficial and detrimental consequences of microglial phagocytosis. Front Cell Neurosci. 7, 6.

Su, Y., Blazey, T.M., Snyder, A.Z., Raichle, M.E., Marcus, D.S., Ances, B.M., Bateman, R.J., Caims, N.J., Aldea, P., Cash, L., Christensen, J.J., Friedrichsen, K. Hornbeck, R.C., Farrar, A.M., Owen, C.J., Mayeux, R., Brickman, A.M., Klunk, W. Price, J.C., Thompson, P.M., Ghetti, B., Saykin, A.J., Sperling, R.A., Johnson, K.A. Schofield, P.R., Buckles, V., Morris, J.C., Benzinger, T.L.S., the Dominantly Inherited Alzheimer Network, 2015. Partial volume correction in quantitative amyloid imaging. Neuroimage 107, 55-64.

Tanaka, T., Ueno, M., Yamashita, T., 2009. Engulfment of axon debris by microglia requires p38 MAPK activity. J. Biol. Chem. 284, 21626-21636.

Thomas, B.A., Erlandsson, K., Modat, M., Thurfjell, L., Vandenberghe, R., Ourselin, S. Hutton, B.F., 2011. The importance of appropriate partial volume correction for PET quantification in Alzheimer's disease. Eur. J. Nucl. Med. Mol. Imaging 38, 1104-1119. 